Published in final edited form as:

Cancer. 2018 June 01; 124(11): 2327-2336. doi:10.1002/cncr.31339.

\title{
Impact of post-transplant lymphoproliferative disorder subtype on survival
}

\author{
Jean L. Koff, MD'1 Jing-Xia Li ${ }^{1,2}$, Xinyan Zhang, PhD, MPH ${ }^{3}$, Jeffrey M. Switchenko, PhD, \\ MS $^{3}$, Christopher R. Flowers, MD, MS ${ }^{1}$, and Edmund K. Waller, MD, PhD, FACP1 \\ ${ }^{1}$ Hematology and Medical Oncology, Winship Cancer Institute, Emory University, Atlanta, GA, \\ United States \\ ${ }^{2}$ Hematology, The First Affiliated Hospital, Sun Yat-sen University, Guangzhou, China \\ ${ }^{3}$ Biostatistics and Bioinformatics, Winship Cancer Institute, Emory University, Atlanta, GA, United \\ States
}

\begin{abstract}
Introduction-Post-transplant lymphoproliferative disease (PTLD) is a life-threatening complication of solid organ transplantation (SOT). Histologic heterogeneity and lack of treatment standards have made evaluating clinical outcomes in specific patient populations difficult. This systematic literature review investigates the impact of PTLD histologic subtype on survival in a large dataset.
\end{abstract}

\begin{abstract}
Methods-Case series were identified on PubMed using the search terms "post-transplant lymphoproliferative disorder/disease," "PTLD", and "solid organ transplantation," with additional publications identified through reference lists. Patient characteristics, immunosuppressive regimen, treatment, survival, and follow-up time of 265 cases were extracted from 94 articles and combined with 11 cases from our institution. Patients with recorded subtype were included in Kaplan-Meier survival analysis $(\mathrm{n}=234)$. Cox proportional hazard regression analyses identified predictors of overall survival (OS) for each subtype and B-cell subgroup.
\end{abstract}

Results-OS differed significantly between monomorphic T-cell neoplasms (median 9 months) and polymorphic, monomorphic B-cell, and Hodgkin-type neoplasms, for which median OS was not reached $(\mathrm{p}=0.0001)$. Significant differences in OS among B-subgroups were not detected, but there was a trend towards decreased survival in Burkitt-type PTLD. Kidney transplant and reduction of immunosuppression were associated with increased OS in multivariable analysis in B-cell neoplasms. Immunosuppression with azathioprine was associated with decreased OS in Tcell neoplasms, while radiotherapy was associated with improved OS in that subtype.

Corresponding author: Jean L. Koff, MD, jkoff@emory.edu, 1365 Clifton Rd NE, Building B, Suite 4300, Atlanta, GA 30322, Telephone: 404.778.5210, Fax: 404.778.3366.

Conflicts of interest: There are no conflicts of interest disclosures from any authors.

Author contributions: All authors contributed extensively to the work presented in this paper. EKW and JLK designed the study. CRF contributed conceptual advice. JL and JLK conducted systematic literature review. JL, XZ, and JMS performed statistical analyses. JLK and JL interpreted the data and prepared the manuscript, edited by EKW and CRF. 
Conclusions-Histologic subtype represents an important prognostic factor in PTLD, with Tcell neoplasms exhibiting very poor OS. Possible lower survival in certain subsets of B-cell PTLD should be explored further and suggests the need for subtype-specific therapies to improve outcomes.

\section{Keywords}

post-transplant lymphoproliferative disorder; survival; immunosuppression; B-cell neoplasms; Tcell neoplasms

\section{Introduction}

Post-transplant lymphoproliferative disease (PTLD) comprises a group of lymphoid disorders arising in the setting of immunosuppression following solid organ transplantation (SOT) or hematopoietic stem cell transplantation (HSCT). Reported cumulative incidences of PTLD in SOT range from 1 to $20 \%$ in SOT depending on transplanted organ, with peak incidence occurring 30-72 months after transplant ${ }^{1,2}$, and 0.5 to $1.0 \%$ in $\mathrm{HSCT}^{3}$, with peak incidence 2 to 4 months post-transplant ${ }^{3,4}$. Reports of PTLD-related mortality range from $30-60 \%$, making this entity a major life-threatening complication of transplant ${ }^{5}$.

Accordingly, much work has been devoted to deciphering an etiologic framework for this complex disease in terms of both biology and epidemiology. However, the relative rarity of the disease has made evaluating clinical outcomes in specific patient populations difficult, since many analyses are limited by small sample size.

Morphologically and molecularly heterogeneous, PTLD is broadly categorized into four groups: early (plasmacytic hyperplasia and infectious mononucleosis-like lesions); polymorphic; monomorphic, which includes all T-cell/natural killer (NK) cell neoplasms as well as most B-cell neoplasms; and classical Hodgkin lymphoma ${ }^{6}$. Treatment of PTLD often varies according to subtype and from institution to institution, ${ }^{7}$ and is complicated by a difficult balance between the risks and benefits of reducing immunosuppression and the intensity of chemo-immunotherapy, which help to eradicate the malignancy but may increase risks of treatment-related toxicity and organ rejection. For patients with CD20+ monomorphic B-cell PTLD who do not respond to reduction of immunosuppression (RI) alone, the monoclonal CD20 antibody rituximab has emerged in the last 15 years as a viable therapeutic option when given alone, ${ }^{8-10}$ in combination with, ${ }^{11,12}$ or followed by chemotherapy with regimens such as cyclophosphamide, doxorubicin, vincristine and prednisone (CHOP). ${ }^{13}$ Studies of PTLD outcomes in the post-rituximab era have largely been limited to the phase II trials investigating these regimens, and single-center experiences. ${ }^{14,15}$

Although it seems reasonable that response to therapeutic agents and survival in certain monomorphic PTLD subgroups would mirror that of the de novo lymphoid malignancies they resemble (e.g., Burkitt or diffuse large B-cell lymphoma), data on subtype-specific outcomes remains limited, perhaps in part because such subgroup categorization may not be captured in epidemiologic datasets. Thus, we conducted a systematic literature review that 
focused on detailed case series to investigate the impact of histologic subtype on survival in PTLD after SOT.

\section{Methods}

\section{Systematic literature review}

Case reports and series were identified on PubMed using the search terms "post-transplant lymphoproliferative disorder/disease," "PTLD", and "solid organ transplantation," generating a pool of 194 articles. Additional manuscripts identified through the reference lists of these papers were incorporated into the pool, which were assessed by inclusion criteria for suitability as shown in Figure 1. Inclusion criteria were: 1) articles published in English between January 1, 1974 and July 1, 2016 with pathology diagnosis and survival details; 2) adult cases following SOT.

A total of 306 cases were extracted from 94 articles (listed in Supplemental Table) and combined with 11 cases from our institution. Data abstracted included patient characteristics (age and gender), transplanted organ(s), immunosuppressive regimen, histologic subtype, initial PTLD treatment, response to treatment, time interval between transplant and PTLD diagnosis (in months), time interval between PTLD diagnosis and death (in months), and length of follow-up after PTLD diagnosis (in months). Based upon the description in the primary publication, histologic subtypes were coded as polymorphic, monomorphic B-cell, monomorphic T-cell, or Hodgkin-like (HL); there were no early PTLD cases identified in our review of published cases. Monomorphic B-cell neoplasms were also further subdivided into four subgroups: diffuse large B-cell lymphoma (DLBCL), Burkitt lymphoma, plasmacytoid, and other/unspecified.

\section{Statistical analysis}

Univariate association of each covariate described above with each of four major subtypes (polymorphic, monomorphic B-cell, monomorphic T-cell, and HL neoplasms) and with four subgroups of B-cell neoplasms was assessed using the chi-square test for categorical covariates and ANOVA for numerical covariates.

Patients with recorded subtype information and reported survival on follow-up were included in survival analysis $(\mathrm{n}=234)$. Univariate and multivariable Cox proportional hazard regression analyses were performed to identify predictors of overall survival (OS) for each subtype and B-cell subgroup. OS was defined as time from diagnosis to death or last followup, where those still alive were censored at last follow-up. The multivariable model was fit by a backward variable selection method, applying a level of significance (a) of 0.10 as removal criteria. Survival curves were constructed using the Kaplan-Meier method and compared with the log-rank test. To evaluate the impact of changes in standard therapy over time on survival, we divided cases into eras by year of publication: 1974 to 1999 to reflect the years before widespread rituximab use, and 2000-2016, by which time rituximab was commonly used to treat other B-cell malignancies and the first retrospective analyses of its use in CD20+ PTLD had been reported. ${ }^{16,17}$ 
A level of significance (a) of 0.05 was considered statistically significant. All statistical analyses were conducted using SAS Version 9.4, with SAS macros and software developed at the Biostatistics and Bioinformatics Core at Winship Cancer Institute. ${ }^{18}$

\section{Results}

Table 1 depicts baseline characteristics and treatment information for the entire cohort of cases $(\mathrm{n}=234)$. Of these, $52.1 \%$ were B-cell monomorphic neoplasms (of which DLBCL was the most frequent subtype [ $\mathrm{n}=59,48.3 \%$ of B neoplasms]), $28.6 \%$ were $\mathrm{T}$ monomorphic neoplasms, $10.6 \%$ were HL, and $4.3 \%$ were polymorphic. Compared to other subtypes, HLPTLD patients were younger at diagnosis (mean age of 37, p = 0.001), and among B-cell monomorphic PTLD, Burkitt-type PTLD patients were significantly younger (mean age of $37, \mathrm{p}<0.001$ ), mirroring incidence patterns seen in corresponding de novo lymphoma types. As might be expected, polymorphic cases were more likely to be treated with surgery and less likely to receive chemotherapy than most other subtypes. Compared to other B-cell subgroups, treatment with chemotherapy was significantly more common in Burkitt-type PTLD (92.9\%, p = 0.014). Univariate comparisons of variables between PTLD subtypes and among B-cell subgroups are shown in Tables 2 and 3, respectively.

In survival analysis, OS was significantly different between histological and immunophenotypic subtypes. Monomorphic T-cell neoplasms had worse survival (median 9 months), while median OS was not reached for polymorphic, monomorphic B-cell, and HL PTLD (log-rank p=0.0001, Figure 2a). Two-year OS with 95\% confidence intervals for each subtype were as follows: $35 \%$ (23.4-46.9\%) in monomorphic T cell, $70.0 \%(32.9-89.2 \%)$ in polymorphic, 60.6\% (50.6-69.2\%) in monomorphic B cell, and 76.5\% (51.9-89.7\%) in HL neoplasms. Significant differences in OS among B-subgroups were not detected, but there was a trend towards decreased survival in Burkitt-type PTLD (Figure 2b). We found that while survival outcomes appeared to improve for all histologic subtypes in the "modern," post-rituximab period, prognosis for T-cell neoplasms remained poor regardless of treatment era (Figure 3). The greatest improvement in survival was seen in Hodgkin-like subtype: 2year overall OS was $100 \%$ in the 2000-2016 era, compared to $67.5 \%$ (38.4-85.1\%) in the 1974-1999 era. The small number of total monomorphic B-cell cases in the pre-rituximab era $(n=23)$ precluded us from comparing outcomes for B-cell subgroups between treatment eras. As shown in Table 4, kidney transplant and treatment with reduction of immunosuppression were associated with increased OS in multivariable analysis in B-cell neoplasms. Immunosuppression with azathioprine was associated with decreased OS in Tcell neoplasms, while treatment with radiotherapy was associated with improved OS in that subtype. (Table 5).

\section{Discussion}

To investigate outcomes in specific PTLD subtypes, our systematic review sought to increase representation of relatively rare subgroups by aggregating clinical and survival data from published case series. This unconventional approach helped to mitigate the dual problem of small sample size, which often limits analyses derived from single-center experiences, and the lack of pathologic specificity that plagues many larger epidemiologic 
databases. The potential limitations of our study include those inherent to carrying out any systematic review, including publication bias and study selection bias. Specifically, we suspect that the higher-than-expected frequency of monomorphic T-cell neoplasms in our dataset may be due to publication bias towards presentation of more unusual cases. We performed a comprehensive systematic review of the literature including a search of relevant publications cited by each source to address these issues where possible. Given our focus on case series, our approach was also limited by publications that lacked central pathology review or provided inadequate data for inclusion in survival analyses. Specifically, missing or incomplete data on EBV association (27\%) hindered our ability to reliably assess this as a factor in subtype-specific survival. Comprehensive prospectively collected data in large cohort studies that include patients with PTLD are needed to meaningfully capture these clinical factors and assess their impact on survival. Nevertheless, our approach represents a large retrospective dataset assembled to examine PTLD subtype-specific clinical factors, treatment patterns, and outcomes, and provides provocative data for future investigation.

Our finding that patients with monomorphic T-cell PTLD exhibited poor survival compared to other subtypes is supported by a recent meta-analysis that examined T-cell PTLD alone. In that study, which included pediatric cases, median OS for all 156 cases was only 6 months, with subgroup-specific (i.e., hepatosplenic, anaplastic, primary cutaneous anaplastic, or peripheral $\mathrm{T}$ cell lymphoma, not otherwise specified) OS ranging from 2 to 18 months. ${ }^{19} \mathrm{~T}$-cell subtype was also found to be a negative prognostic factor for OS in a Swedish study of 135 PTLD cases (of which 15 were T-cell neoplasms), with a hazard ratio (HR) of $3.52(\mathrm{p}<0.001)$ in multivariable Cox regression analysis. ${ }^{20}$

Although not statistically significant, a trend towards decreased survival in Burkitt-type monomorphic B-cell PTLD seen in our study merits investigation in larger datasets. The concept that Burkitt-type PTLD should be considered a discrete entity within B-cell PTLD, with clinicopathologic features that also distinguish it from sporadic, endemic, and immunodeficiency-associated Burkitt lymphomas, has been posited in the pediatric setting.

${ }^{21}$ Interestingly, although cases in that particular series uniformly exhibited aggressive clinical behavior, none of the patients who died during follow-up exhibited evidence of unresponsive and/or progressive disease at time of death.

\section{Potential factors underlying subtype-specific outcomes in PTLD}

Given that 50 to $70 \%$ of PTLDs are Epstein-Barr virus (EBV)-related, current understanding of PTLD development uses the model of malignant transformation of EBV-infected B-cells in the setting of reduced T-cell control that results from immunocompromise and/or immunosuppression ${ }^{2,22-24}$. However, such models do not account for the significant proportion of PTLD cases in which the role of EBV is not readily apparent, such as in neoplasms arising from T- or NK-cells-although presence of EBV DNA in T-cell PTLD tumor cells has been described. ${ }^{25}$ Disparities in timing of peak incidence between SOT and HSCT suggest differences in etiologic pathways as well. In fact, typical SOT-related PTLDs develop from recipient cells, while most HSCT-associated PTLDs arise from donor cells, further adding to the complexity of characterizing oncogenesis in this heterogeneous disease. 26-28 
Perhaps unsurprisingly, other major risk factors for PTLD development relate to modulations of the immune milieu. Reported risk factors in SOT include transplantation of lymphoid-rich organs such as lung and intestine, and transplantation from EBV-seropositive donor to EBV-seronegative recipient, whereas risk factors in HSCT include T cell-depleted graft, HLA mismatch, and primary immunodeficiency ${ }^{26,29}$. Estimation of the intensity of pharmacological immunosuppression between individual patients and across clinical SOT protocols is challenging, and associations between immunosuppression and PTLD have been derived only indirectly from registry analyses and observational studies. ${ }^{30}$ In both SOT and HSCT, T-cell-depleting agents such as anti-thymocyte globulin (ATG) and the anti-CD3 monoclonal antibody OKT3 are associated with PTLD development. ${ }^{15,31-33}$ Interestingly, increased PTLD risk has not been observed with alemtuzumab, ${ }^{34}$ a monoclonal antibody directed against the CD52 marker present on both B- and T-cells, leading some to speculate that that drug's additional B-cell-depleting effect could serve to reduce the risk of B-cell PTLD. In the maintenance setting, the calcineurin inhibitor tacrolimus has been associated with increased PTLD risk in several large studies, $31,32,35,36$ but mycophenolate mofetil does not appear to exert this same effect. ${ }^{15,35,37-40}$ Although the mechanisms underlying these differences in risk remain unclear, it is worth noting that mycophenolate acts to suppress both B- and T-cells, while tacrolimus' immunosuppressive effect is more T-cell-specific.

Conversely, prophylactic anti-viral use following transplantation may reduce risk of PTLD development up to $83 \%$, depending on the anti-viral agent ${ }^{41}$. The combination of monitoring serum EBV-DNA load and adjusting immunosuppression is similarly associated with lower incidence of PTLD after SOT ${ }^{42,43}$. Indeed, RI is a frequently used initial approach for PTLD that can reconstitute cytotoxic T-cell function against uncontrolled lymphoproliferation, with response rates reported as high as $45 \%{ }^{44}$.

It is difficult to evaluate the role of individual immunosuppressive agents and RI strategies in PTLD risk and outcomes, especially since transplant patients may receive a variety of drug combinations during induction, maintenance, and/or treatment for graft rejection. ${ }^{45}$ Although the relatively small sample size in our multiple variable regression models limits their power, our results indicate that RI is associated with improved OS in B-cell but not Tcell PTLD. These findings suggest subtype-specific differences in susceptibility to cytotoxic T-cell effects, which may in turn reflect etiologic differences in pathogenesis (e.g., EBVinduced malignant transformation in B-cell but not T-cell neoplasms). Moreover, the association of azathioprine with decreased OS in T-cell PTLD may implicate particular immunosuppressive agents as barriers to eradication of certain PTLD subtypes. Our findings are supported by a large study of 230 cases from the French Registry of PTLD occurring after renal transplant, which identified azathioprine as a factor associated with poor overall survival, but that study did not show a subtype-specific association or survival differences based on histologic factors. ${ }^{46}$ Although azathioprine's inhibition of purine nucleotide biosynthesis with suppression of DNA and RNA synthesis has been well described, the specific mechanism(s) by which it down-regulates B-cell and T-cell function to induce immunosuppression are less well characterized. In vitro and in vivo data suggest that azathioprine may interfere with CD28 costimulation of alloreactive $\mathrm{T}$ cells by blocking the GTPase Rac1, which results in activation of apoptotic pathways upon CD28-mediated signaling. ${ }^{47}$ Furthermore, azathioprine may preferentially induce apoptosis in CD45RO 
memory T-cells through suppression of expression of the anti-apoptotic protein BCL-XL, thus offering a possible explanation for azathioprine's ability to induce tolerance to foreign antigen (e.g., of an organ graft). However, it remains unclear how elimination of this T-cell population might contribute to worse prognosis in T-cell PTLD. To answer this and other complex questions surrounding the impact of immunosuppressive regimen on subtypespecific outcomes, prospective epidemiologic studies are needed to link detailed clinical information with data from biologic specimens, including tumor tissue and longitudinally collected peripheral blood samples.

As in de novo lymphoid malignancies, subtype-specific survival in PTLD may also depend on response to immunochemotherapeutic regimens. Importantly, better survival in B-cell PTLDs may in part reflect improved treatment responses seen with rituximab treatment, unavailable to PTLD subtypes without CD20 expression. In a retrospective multicenter analysis of 80 SOT-related PTLD patients who received reduction of immunosuppression, those who received concomitant frontline rituximab-based therapy experienced improved 3year progression free survival and OS (70\% and 73\%, respectively), compared to those who did not (21\% and 33\%). ${ }^{48}$ However, that study did not differentiate between subtypes of monomorphic PTLD. Three prospective phase II trials provided evidence that rituximab monotherapy has efficacy in CD20-positive PTLD, but all of these demonstrated relatively short time-to-progression intervals. ${ }^{8-10}$ Accordingly, the prospective multicenter PTLD-1 trial investigated a sequential treatment approach with 4 weeks of rituximab followed by chemotherapy with CHOP in SOT-related CD20-positive PTLD failing to respond to RI. ${ }^{49}$ Sixty percent of patients (42/70) achieved either a complete response (CR) or partial response (PR) after rituximab monotherapy, with response rate improving to 90\% (53/59) with CR rate of $68 \%$ upon completion of sequential therapy. Median survival in responders was not reached at time of analysis, and response to rituximab prior to chemotherapy was predictive of time to progression and OS. EBV-associated disease was also found to be favorable in terms of time to progression, further emphasizing rituximab's role in PTLD outcomes.

Although we used the advent of widespread rituximab use as a cut-point to analyze changes in subtype-specific survival over time, the most striking difference in survival between treatment eras occurred among HL-PTLD, a CD20-negative subtype that does not benefit from rituximab treatment. Sample sizes are admittedly small, but it is unclear what factors led to this apparent increase over time. Notably, none of the articles reported treatment with newer agents such as the CD30-targeting antibody-drug conjugate brentuximab vedotin. It is possible that this may reflect a shift in supportive care or diagnostic knowledge, but it may also lend support to the findings of Rosenberg et al. that patients with HL-PTLD who received chemotherapeutic regimens traditionally used in Hodgkin lymphoma experienced improved survival. ${ }^{50}$ That recent retrospective analysis utilized the Scientific Registry for Transplant Recipients (SRTR) and Surveillance Epidemiology and End Results (SEER) databases to compare outcomes between HL-PTLD and de novo Hodgkin lymphoma, highlighting several interesting ideas pertaining to PTLD subtype. For HL-PTLD patients, receipt of any chemotherapy was associated with decreased hazard of death $(\mathrm{HR}=0.36, \mathrm{p}<$ 0.001), and patients who received nontraditional Hodgkin regimens had increased hazard of death (adjusted HR $=2.01, \mathrm{p}=0.04$ ) compared to those who received Hodgkin-specific 
chemotherapy regimens. To account for the fact that patients with HL-PTLD would be more likely to die of non-lymphoma causes than patients without prior SOT (e.g., graft failure), the authors calculated cumulative incidence curves of lymphoma-specific mortality, treating other causes of death as competing risks. HL-PTLD patients exhibited a 5-year lymphomaspecific mortality rate of $23 \%$, compared to $13 \%$ for patients with de novo (p <0.001). Taken together, these findings suggest that subtype-directed therapy remains an important consideration in PTLD, but lymphoma-specific survival for HL PTLD remains worse for than its de novo counterpart. Additional studies utilizing large datasets are needed to determine whether such conclusions hold true for other rare subgroups of monomorphic PTLD. Improved understanding of the underlying differences in the pathogenesis of PTLD subtypes and outcomes for subtype-directed therapies compared to their de novo counterparts will be necessary to tailor appropriate treatments for each subtype.

\section{Conclusions}

Our work lends credence to the emerging concept that PTLD subtype carries major implications in terms of both prognosis and treatment options. Importantly, we show that patients with monomorphic T-cell PTLD suffer inferior survival compared to all other subtypes. Possible lower survival in certain subsets of monomorphic B-cell PTLD should also be explored further and suggests the need for subtype-specific therapies to improve outcomes. Multi-institution collaborative efforts could evaluate these associations in a larger number of cases with the necessary level of histologic discrimination. In general, performing meaningful studies of subtype-specific disparities in PTLD in the future will require collection of biological specimens as well as clinical data to examine the effect of PTLD subtype on differences in disease presentation, treatment selection, and patient outcomes.

\section{Supplementary Material}

Refer to Web version on PubMed Central for supplementary material.

\section{Acknowledgments}

Funding: Research reported in this publication was supported in part by the Biostatistics and Bioinformatics Shared Resource of Winship Cancer Institute of Emory University and NIH/NCI under award number P30CA138292. The content is solely the responsibility of the authors and does not necessarily represent the official views of the National Institutes of Health.

\section{References}

1. Gottschalk S, Rooney CM, Heslop HE. Post-transplant lymphoproliferative disorders. Annu Rev Med. 2005; 56:29-44. [PubMed: 15660500]

2. Jagadeesh D, Woda BA, Draper J, Evens AM. Post transplant lymphoproliferative disorders: risk, classification, and therapeutic recommendations. Curr Treat Options Oncol. 2012; 13:122-36. [PubMed: 22241590]

3. Bhatia S, Ramsay NK, Steinbuch M, et al. Malignant neoplasms following bone marrow transplantation. Blood. 1996; 87:3633-9. [PubMed: 8611687]

4. Novoa-Takara L, Perkins SL, Qi D, et al. Histogenetic phenotypes of B cells in posttransplant lymphoproliferative disorders by immunohistochemical analysis correlate with transplant type: solid organ vs hematopoietic stem cell transplantation. Am J Clin Pathol. 2005; 123:104-12. [PubMed: 15762285] 
5. Mucha K, Foroncewicz B, Ziarkiewicz-Wroblewska B, Krawczyk M, Lerut J, Paczek L. Posttransplant lymphoproliferative disorder in view of the new WHO classification: a more rational approach to a protean disease? Nephrol Dial Transplant. 2010; 25:2089-98. [PubMed: 20576725]

6. Swerdlow SH, International Agency for Research on Cancer., World Health Organization. WHO classification of tumours of haematopoietic and lymphoid tissues. 4. Lyon, France: International Agency for Research on Cancer; 2008.

7. Allen U, Hebert D, Moore D, Dror Y, Wasfy S, Canadian PSG. Epstein-Barr virus-related posttransplant lymphoproliferative disease in solid organ transplant recipients, 1988-97: a Canadian multi-centre experience. Pediatr Transplant. 2001; 5:198-203. [PubMed: 11422823]

8. Oertel SH, Verschuuren E, Reinke P, et al. Effect of anti-CD 20 antibody rituximab in patients with post-transplant lymphoproliferative disorder (PTLD). Am J Transplant. 2005; 5:2901-6. [PubMed: 16303003]

9. Choquet S, Leblond V, Herbrecht R, et al. Efficacy and safety of rituximab in B-cell posttransplantation lymphoproliferative disorders: results of a prospective multicenter phase 2 study. Blood. 2006; 107:3053-7. [PubMed: 16254143]

10. Gonzalez-Barca E, Domingo-Domenech E, Capote FJ, et al. Prospective phase II trial of extended treatment with rituximab in patients with B-cell post-transplant lymphoproliferative disease. Haematologica. 2007; 92:1489-94. [PubMed: 18024397]

11. Choquet S, Trappe R, Leblond V, Jager U, Davi F, Oertel S. CHOP-21 for the treatment of posttransplant lymphoproliferative disorders (PTLD) following solid organ transplantation. Haematologica. 2007; 92:273-4. [PubMed: 17296588]

12. Elstrom RL, Andreadis C, Aqui NA, et al. Treatment of PTLD with rituximab or chemotherapy. Am J Transplant. 2006; 6:569-76. [PubMed: 16468968]

13. Trappe R, Oertel S, Leblond V, et al. Sequential treatment with rituximab followed by CHOP chemotherapy in adult B-cell post-transplant lymphoproliferative disorder (PTLD): the prospective international multicentre phase 2 PTLD-1 trial. The Lancet Oncology. 2012; 13:196-206. [PubMed: 22173060]

14. Morton M, Coupes B, Roberts SA, et al. Epidemiology of posttransplantation lymphoproliferative disorder in adult renal transplant recipients. Transplantation. 2013; 95:470-8. [PubMed: 23222821]

15. Caillard S, Lamy FX, Quelen C, et al. Epidemiology of posttransplant lymphoproliferative disorders in adult kidney and kidney pancreas recipients: report of the French registry and analysis of subgroups of lymphomas. Am J Transplant. 2012; 12:682-93. [PubMed: 22226336]

16. Milpied N, Vasseur B, Parquet N, et al. Humanized anti-CD20 monoclonal antibody (Rituximab) in post transplant B-lymphoproliferative disorder: a retrospective analysis on 32 patients. Ann Oncol. 2000; 11(Suppl 1):113-6.

17. Oertel SH, Anagnostopoulos I, Bechstein WO, Liehr H, Riess HB. Treatment of posttransplant lymphoproliferative disorder with the anti-CD20 monoclonal antibody rituximab alone in an adult after liver transplantation: a new drug in therapy of patients with posttransplant lymphoproliferative disorder after solid organ transplantation? Transplantation. 2000; 69:430-2. [PubMed: 10706055]

18. Nickleach D, Liu Y, Shrewsberry A, Ogan K, Kim S, Wang Z. SAS® macros to conduct common biostatistical analyses and generate reports. SESUG 2013: The Proceeding of the SouthEast SAS User Group. 2013

19. Herreman A, Dierickx D, Morscio J, et al. Clinicopathological characteristics of posttransplant lymphoproliferative disorders of T-cell origin: single-center series of nine cases and meta-analysis of 147 reported cases. Leuk Lymphoma. 2013; 54:2190-9. [PubMed: 23402267]

20. Kinch A, Baecklund E, Backlin C, et al. A population-based study of 135 lymphomas after solid organ transplantation: The role of Epstein-Barr virus, hepatitis $\mathrm{C}$ and diffuse large B-cell lymphoma subtype in clinical presentation and survival. Acta Oncol. 2014; 53:669-79. [PubMed: 24164103]

21. Picarsic J, Jaffe R, Mazariegos G, et al. Post-transplant Burkitt lymphoma is a more aggressive and distinct form of post-transplant lymphoproliferative disorder. Cancer. 2011; 117:4540-50.

[PubMed: 21446044] 
22. Evens AM, Roy R, Sterrenberg D, Moll MZ, Chadburn A, Gordon LI. Post-transplantation lymphoproliferative disorders: diagnosis, prognosis, and current approaches to therapy. Curr Oncol Rep. 2010; 12:383-94. [PubMed: 20963522]

23. Luskin MR, Heil DS, Tan KS, et al. The Impact of EBV Status on Characteristics and Outcomes of Posttransplantation Lymphoproliferative Disorder. Am J Transplant. 2015; 15:2665-73. [PubMed: 25988622]

24. Nourse JP, Jones K, Gandhi MK. Epstein-Barr Virus-related post-transplant lymphoproliferative disorders: pathogenetic insights for targeted therapy. Am J Transplant. 2011; 11:888-95. [PubMed: 21521464]

25. Waller E, Ziemianska M, Bangs C, Cleary M, Weissman I, Kamel O. Characterization of posttransplant lymphomas that express T-cell- associated markers: immunophenotypes, molecular genetics, cytogenetics, and heterotransplantation in severe combined immunodeficient mice. Blood. 1993; 82:247-61. [PubMed: 8100721]

26. Loren AW, Porter DL, Stadtmauer EA, Tsai DE. Post-transplant lymphoproliferative disorder: a review. Bone Marrow Transplant. 2003; 31:145-55. [PubMed: 12621474]

27. Capello D, Rasi S, Oreste P, et al. Molecular characterization of post-transplant lymphoproliferative disorders of donor origin occurring in liver transplant recipients. J Pathol. 2009; 218:478-86. [PubMed: 19391128]

28. Ruf S, Moser O, Wossmann W, Kreyenberg H, Wagner HJ. Examining the origin of posttransplant lymphoproliferative disorder in a patient after a second allogeneic hematopoeitic stem cell transplantation for relapsed BCR-ABL positive acute lymphoblastic leukemia. J Pediatr Hematol Oncol. 2011; 33:50-4. [PubMed: 21178708]

29. Green M, Michaels MG. Epstein-Barr virus infection and posttransplant lymphoproliferative disorder. Am J Transplant. 2013; 13(Suppl 3):41-54. quiz. [PubMed: 23347213]

30. San-Juan R, Comoli P, Caillard S, et al. Epstein-Barr virus-related post-transplant lymphoproliferative disorder in solid organ transplant recipients. Clin Microbiol Infect. 2014; 20(Suppl 7):109-18. [PubMed: 24475976]

31. Opelz G, Dohler B. Lymphomas after solid organ transplantation: a collaborative transplant study report. Am J Transplant. 2004; 4:222-30. [PubMed: 14974943]

32. Fernberg P, Edgren G, Adami J, et al. Time trends in risk and risk determinants of non-Hodgkin lymphoma in solid organ transplant recipients. Am J Transplant. 2011; 11:2472-82. [PubMed: 21883909]

33. Duvoux C, Pageaux GP, Vanlemmens C, et al. Risk factors for lymphoproliferative disorders after liver transplantation in adults: an analysis of 480 patients. Transplantation. 2002 ; 74:1103-9. [PubMed: 12438954]

34. Kirk AD, Cherikh WS, Ring M, et al. Dissociation of depletional induction and posttransplant lymphoproliferative disease in kidney recipients treated with alemtuzumab. Am J Transplant. 2007; 7:2619-25. [PubMed: 17868060]

35. Bustami RT, Ojo AO, Wolfe RA, et al. Immunosuppression and the risk of post-transplant malignancy among cadaveric first kidney transplant recipients. Am J Transplant. 2004; 4:87-93. [PubMed: 14678038]

36. Caillard S, Dharnidharka V, Agodoa L, Bohen E, Abbott K. Posttransplant lymphoproliferative disorders after renal transplantation in the United States in era of modern immunosuppression. Transplantation. 2005; 80:1233-43. [PubMed: 16314791]

37. Cherikh WS, Kauffman HM, McBride MA, Maghirang J, Swinnen LJ, Hanto DW. Association of the type of induction immunosuppression with posttransplant lymphoproliferative disorder, graft survival, and patient survival after primary kidney transplantation. Transplantation. 2003; 76:1289-93. [PubMed: 14627905]

38. Sampaio MS, Cho YW, Shah T, Bunnapradist S, Hutchinson IV. Association of immunosuppressive maintenance regimens with posttransplant lymphoproliferative disorder in kidney transplant recipients. Transplantation. 2012; 93:73-81. [PubMed: 22129761]

39. Robson R, Cecka JM, Opelz G, Budde M, Sacks S. Prospective registry-based observational cohort study of the long-term risk of malignancies in renal transplant patients treated with mycophenolate mofetil. Am J Transplant. 2005; 5:2954-60. [PubMed: 16303010] 
40. Funch DP, Ko HH, Travasso J, et al. Posttransplant lymphoproliferative disorder among renal transplant patients in relation to the use of mycophenolate mofetil. Transplantation. 2005; 80:1174-80. [PubMed: 16314782]

41. Funch DP, Walker AM, Schneider G, Ziyadeh NJ, Pescovitz MD. Ganciclovir and acyclovir reduce the risk of post-transplant lymphoproliferative disorder in renal transplant recipients. Am J Transplant. 2005; 5:2894-900. [PubMed: 16303002]

42. Bakker NA, Verschuuren EA, Erasmus ME, et al. Epstein-Barr virus-DNA load monitoring late after lung transplantation: a surrogate marker of the degree of immunosuppression and a safe guide to reduce immunosuppression. Transplantation. 2007; 83:433-8. [PubMed: 17318076]

43. Lee TC, Savoldo B, Rooney CM, et al. Quantitative EBV viral loads and immunosuppression alterations can decrease PTLD incidence in pediatric liver transplant recipients. Am J Transplant. 2005; 5:2222-8. [PubMed: 16095501]

44. Reshef R, Vardhanabhuti S, Luskin MR, et al. Reduction of immunosuppression as initial therapy for posttransplantation lymphoproliferative disorder( bigstar). Am J Transplant. 2011; 11:336-47. [PubMed: 21219573]

45. Birkeland SA, Hamilton-Dutoit S. Is posttransplant lymphoproliferative disorder (PTLD) caused by any specific immunosuppressive drug or by the transplantation per se? Transplantation. 2003; 76:984-8. [PubMed: 14508366]

46. Caillard S, Lelong C, Pessione F, Moulin B, French PWG. Post-transplant lymphoproliferative disorders occurring after renal transplantation in adults: report of 230 cases from the French Registry. Am J Transplant. 2006; 6:2735-42. [PubMed: 17049061]

47. Tiede I, Fritz G, Strand S, et al. CD28-dependent Rac1 activation is the molecular target of azathioprine in primary human CD4(+) T lymphocytes. Journal of Clinical Investigation. 2003; 111:1133-45. [PubMed: 12697733]

48. Evens AM, David KA, Helenowski I, et al. Multicenter Analysis of 80 Solid Organ Transplantation Recipients With Post-Transplantation Lymphoproliferative Disease: Outcomes and Prognostic Factors in the Modern Era. Journal of Clinical Oncology. 2010; 28:1038-46. [PubMed: 20085936]

49. Trappe R, Oertel S, Leblond V, et al. Sequential treatment with rituximab followed by CHOP chemotherapy in adult B-cell post-transplant lymphoproliferative disorder (PTLD): the prospective international multicentre phase 2 PTLD-1 trial. The Lancet Oncology. 13:196-206.

50. Rosenberg AS, Klein AK, Ruthazer R, Evens AM. Hodgkin lymphoma post-transplant lymphoproliferative disorder: A comparative analysis of clinical characteristics, prognosis, and survival. Am J Hematol. 2016; 91:560-5. [PubMed: 26928381] 


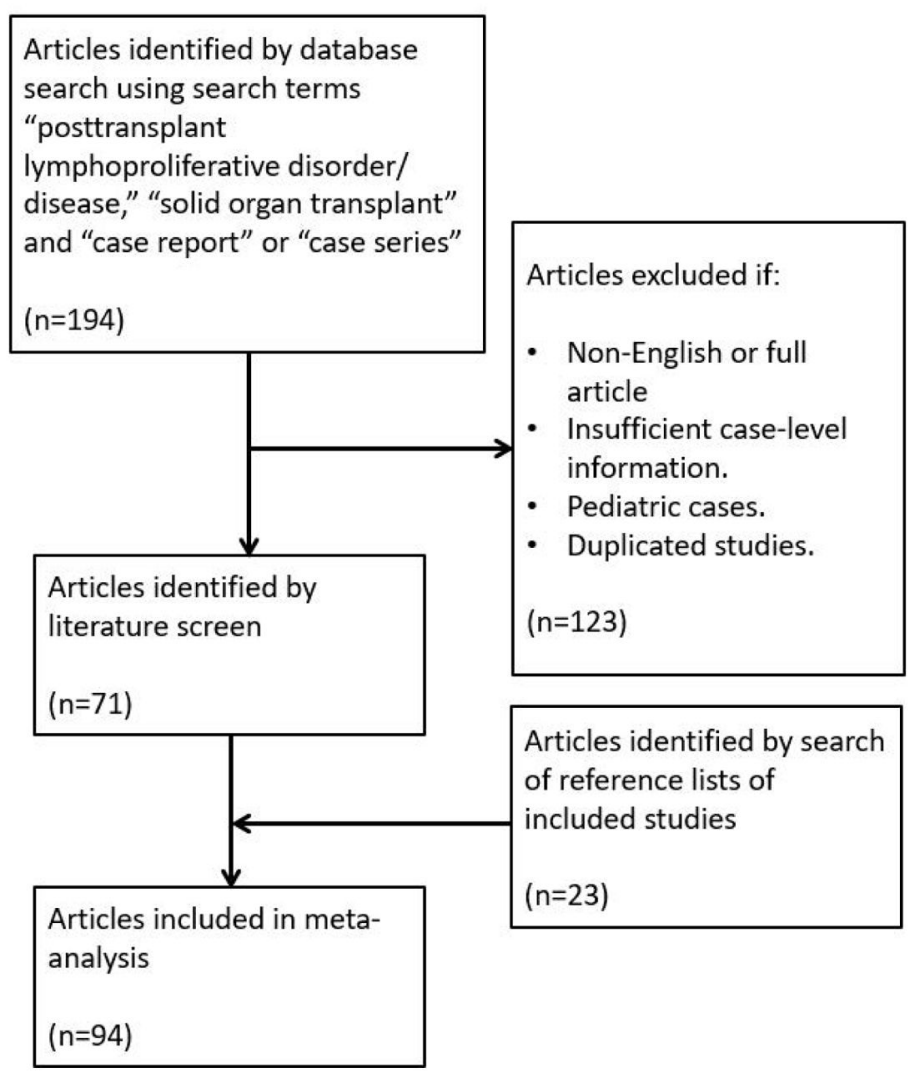

Figure 1.

Selection strategy for articles included in meta-analysis. 
A.

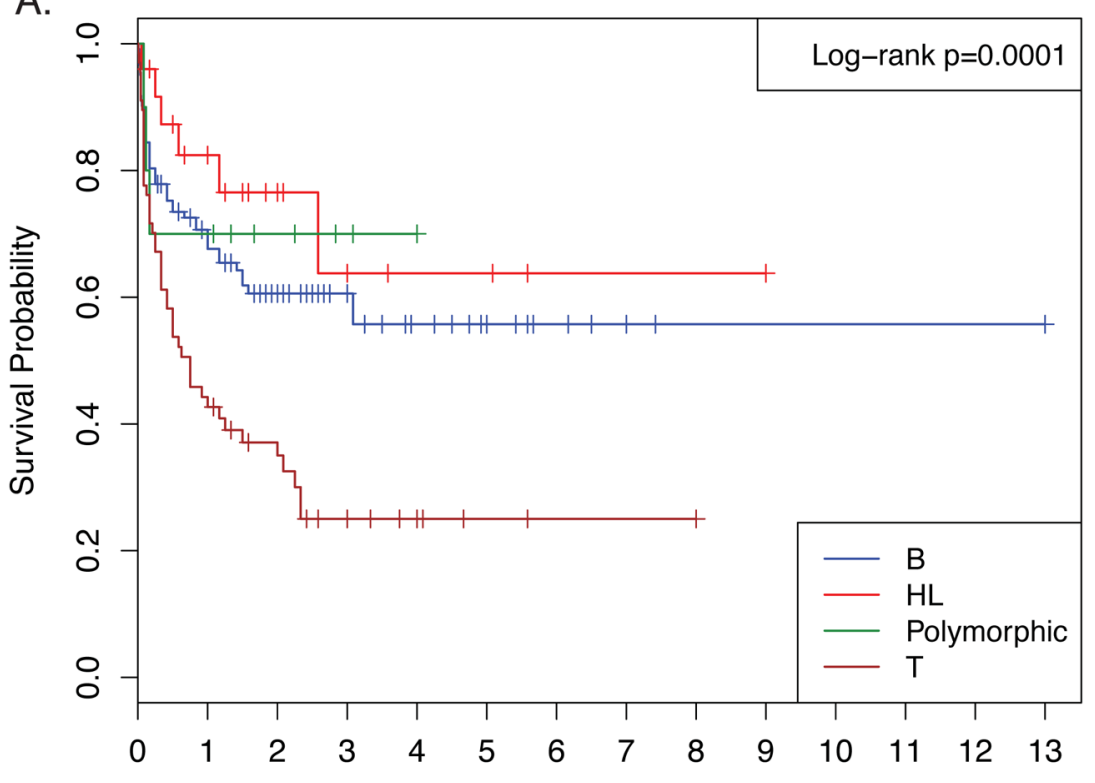

Overall survival (Years)

\begin{tabular}{|c|c|c|c|c|c|c|c|c|c|c|c|c|c|}
\hline & Nun & ber a & $\mathrm{Ris}$ & & & & & & & & & & \\
\hline$B$ & 122 & 70 & 40 & 27 & 16 & 10 & 5 & 3 & 1 & 1 & 1 & 1 & 1 \\
\hline & 25 & 16 & 8 & 5 & 3 & 3 & 1 & 1 & 1 & 1 & 0 & 0 & 0 \\
\hline & 10 & 7 & 4 & 2 & 1 & 0 & 0 & 0 & 0 & 0 & 0 & 0 & 0 \\
\hline & 67 & 28 & 18 & 8 & 5 & 2 & 1 & 1 & 1 & 0 & 0 & 0 & 0 \\
\hline
\end{tabular}

B.

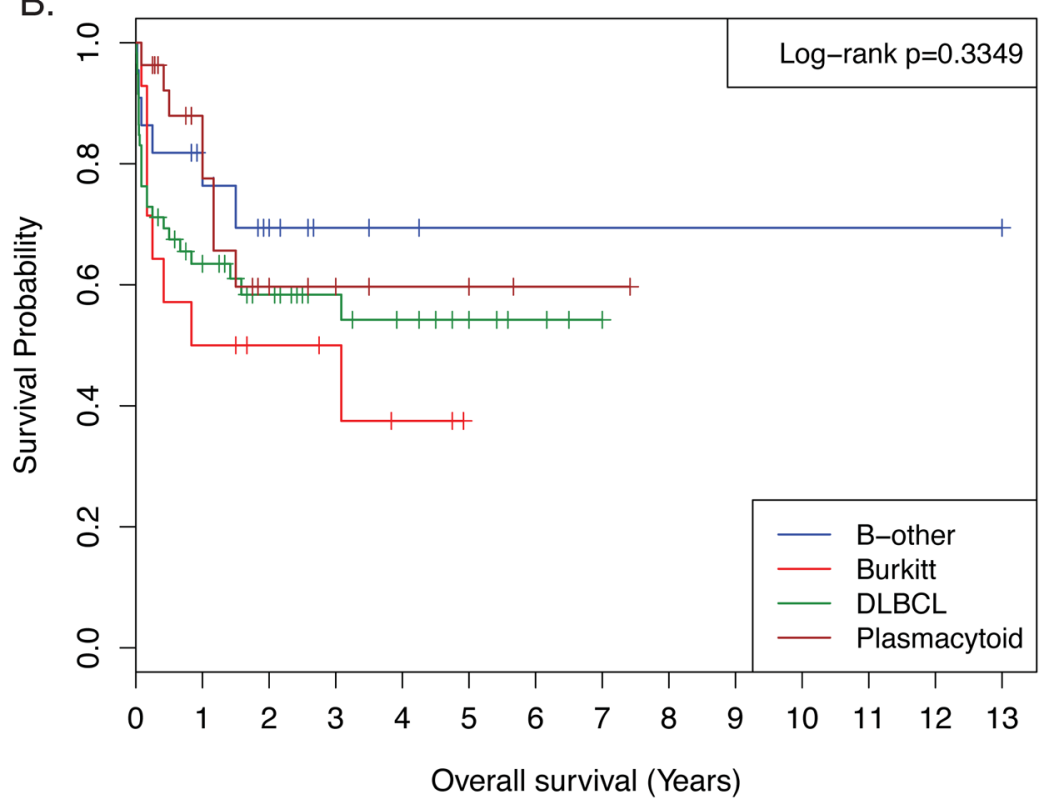

Number at Risk

\begin{tabular}{|c|c|c|c|c|c|c|c|c|c|c|c|c|c|}
\hline B-other & 22 & 15 & 7 & 3 & 2 & 1 & 1 & 1 & 1 & 1 & 1 & 1 & 1 \\
\hline Burkitt & 14 & 7 & 5 & 4 & 2 & 0 & 0 & 0 & 0 & 0 & 0 & 0 & \\
\hline DLBCL & 59 & 31 & 20 & 14 & 9 & 6 & 3 & 1 & 0 & 0 & 0 & 0 & \\
\hline Plasm & 27 & 17 & 8 & 6 & 3 & 3 & 1 & 1 & 0 & 0 & 0 & 0 & 0 \\
\hline
\end{tabular}

Figure 2. Overall survival in patients with PTLD by histologic subtype (A) and in patients with B-cell PTLD by B-cell subgroup (B) 
Abbreviations: B, monomorphic B-cell; T, monomorphic T-cell; DLBCL, diffuse large Bcell lymphoma; HL, Hodgkin-like. 
A.

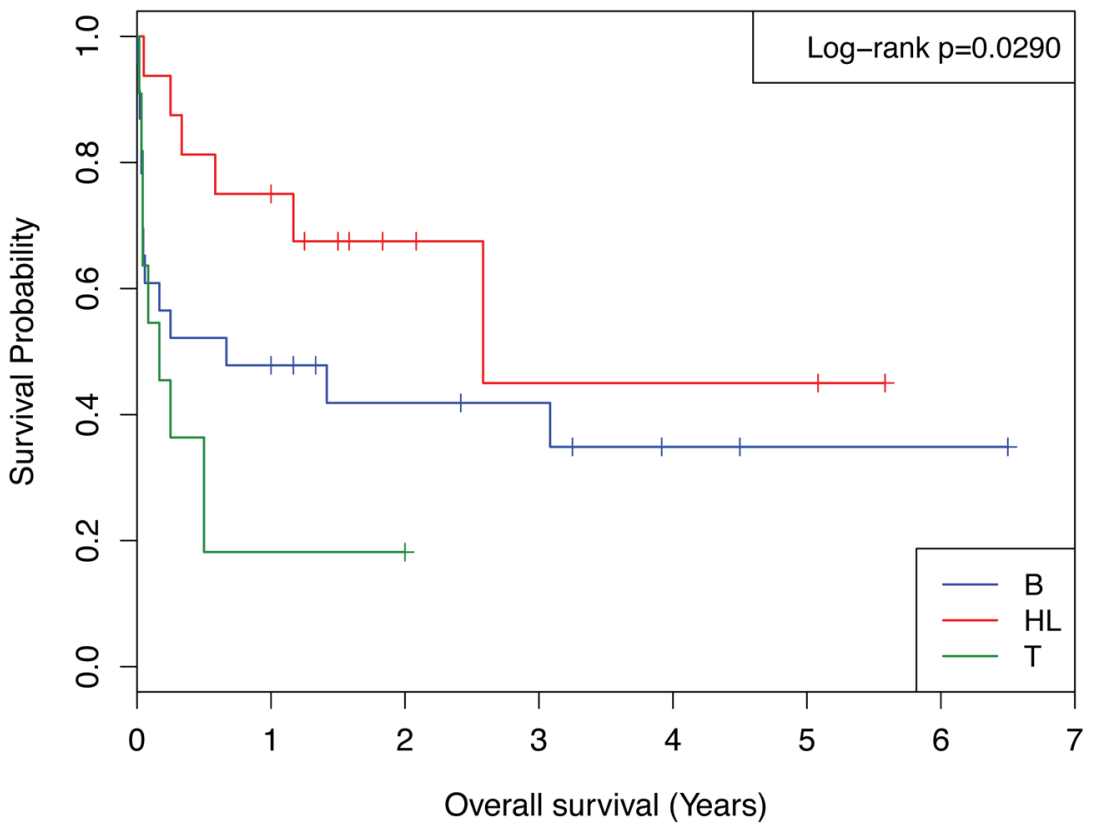

\begin{tabular}{|c|c|c|c|c|c|c|}
\hline $\begin{array}{l}23 \\
16\end{array}$ & $\begin{array}{l}11 \\
12\end{array}$ & 7 & 6 & 2 & $\begin{array}{l}1 \\
0\end{array}$ & $\begin{array}{l}1 \\
0\end{array}$ \\
\hline 11 & 2 & 2 & 0 & 0 & 0 & 0 \\
\hline
\end{tabular}

B.



Figure 3. Overall survival in patients with PTLD by histologic subtype by treatment era

Cancer: Author manuscript; available in PMC 2019 June 01. 
(A) 1974 - 1999. (B) 2000 - 2016. Abbreviations: B, monomorphic B-cell; T, monomorphic T-cell; HL, Hodgkin-like. 


\section{Table 1}

Baseline and treatment characteristics of post-transplant lymphoproliferative disorder (PTLD) cases with available survival data from systematic review. Abbreviations: post-transplant lymphoproliferative disorder (PTLD); diffuse large B-cell lymphoma (DLBCL); standard deviation (SD).

\begin{tabular}{|c|c|}
\hline Variable & Total $n=234(\%)$ \\
\hline \multicolumn{2}{|l|}{ Age at diagnosis } \\
\hline Mean years \pm SD & $47 \pm 14$ \\
\hline \multicolumn{2}{|l|}{ Sex } \\
\hline Female & $59(25.5 \%)$ \\
\hline Male & $172(74.5 \%)$ \\
\hline Missing & 3 \\
\hline \multicolumn{2}{|l|}{ Histologic subtype } \\
\hline B-cell monomorphic & $122(52.1 \%)$ \\
\hline T-cell monomorphic & $67(28.6 \%)$ \\
\hline Hodgkin-type & $25(10.7 \%)$ \\
\hline Polymorphic & $10(4.3 \%)$ \\
\hline Not otherwise specified & $10(4.3 \%)$ \\
\hline B-cell monomorphic subgroups & $n=122$ \\
\hline DLBCL & $59(48.4 \%)$ \\
\hline Burkitt lymphoma & $14(11.5 \%)$ \\
\hline Plasmacytoid & $27(22.1 \%)$ \\
\hline Other/unspecified & $22(18 \%)$ \\
\hline \multicolumn{2}{|l|}{ Transplanted organ(s) } \\
\hline Heart or Heart-Lung & $51(21.8 \%)$ \\
\hline Kidney or Kidney-Pancreas & $130(55.6 \%)$ \\
\hline Lung or Small Bowel & $14(6 \%)$ \\
\hline Liver or Liver-Kidney or Liver-Small Bowel & $39(16.7 \%)$ \\
\hline \multicolumn{2}{|l|}{ Immunosuppressive regimen at diagnosis } \\
\hline \multicolumn{2}{|l|}{ Calcineurin inhibitors } \\
\hline None & $11(0.5 \%)$ \\
\hline Cyclosporine & $157(71.4 \%)$ \\
\hline Tacrolimus & $50(22.7 \%)$ \\
\hline Cyclosporine and tacrolimus & $2(0.9 \%)$ \\
\hline Missing & 14 \\
\hline \multicolumn{2}{|l|}{ Anti-metabolites } \\
\hline None & $51(23.1 \%)$ \\
\hline Azathioprine & $106(48 \%)$ \\
\hline Mycophenolate mofetil & $64(29)$ \\
\hline Missing & 13 \\
\hline
\end{tabular}









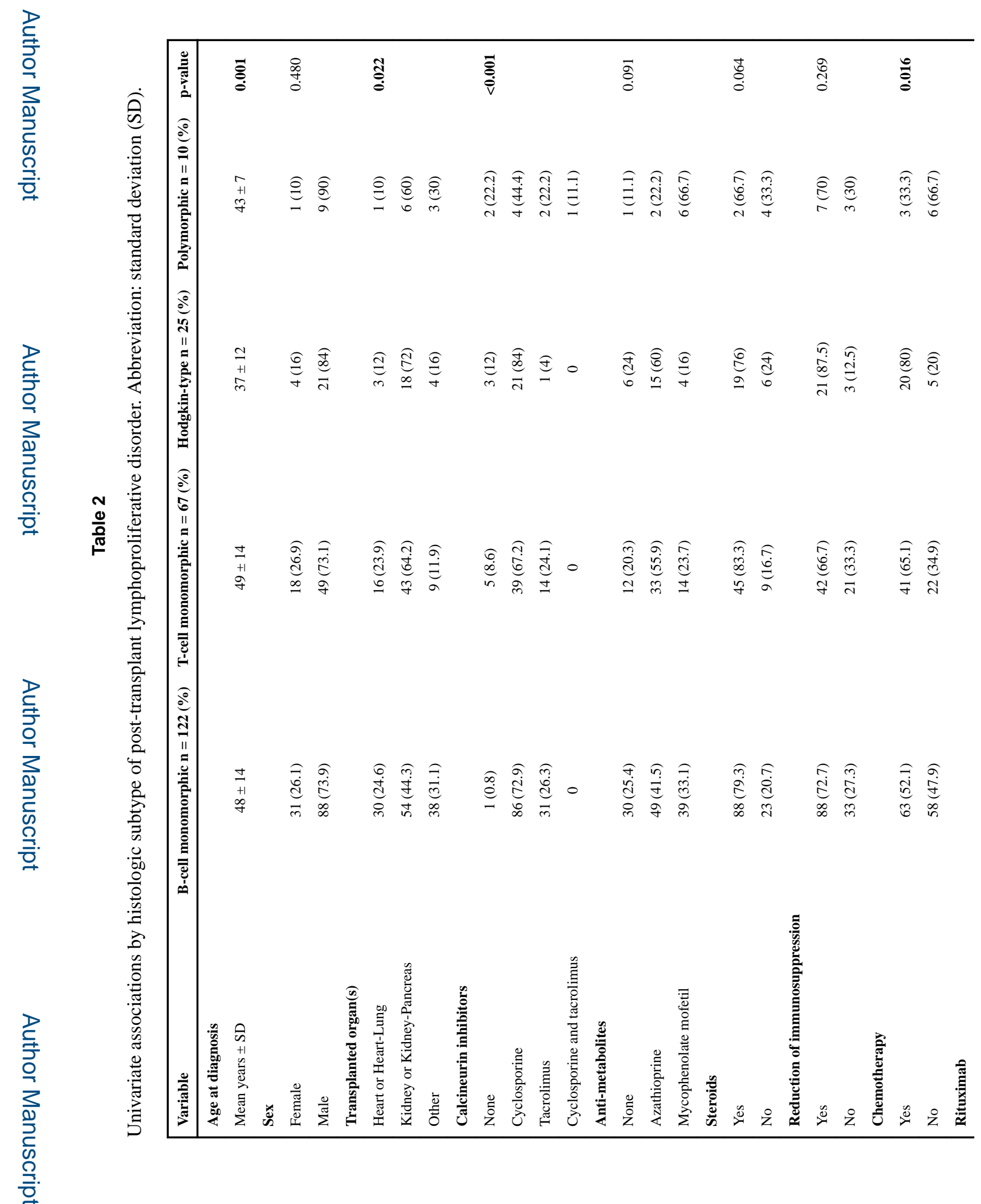

Cancer. Author manuscript; available in PMC 2019 June 01. 
Koff et al.

Page 20

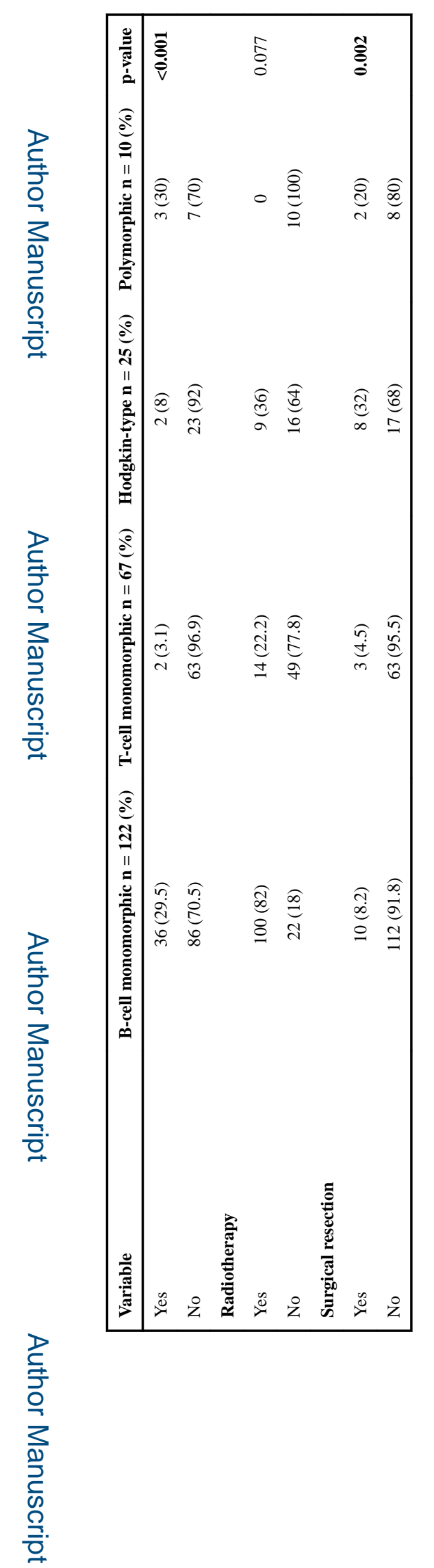

Cancer: Author manuscript; available in PMC 2019 June 01. 




Cancer. Author manuscript; available in PMC 2019 June 01. 
Koff et al.

Page 22

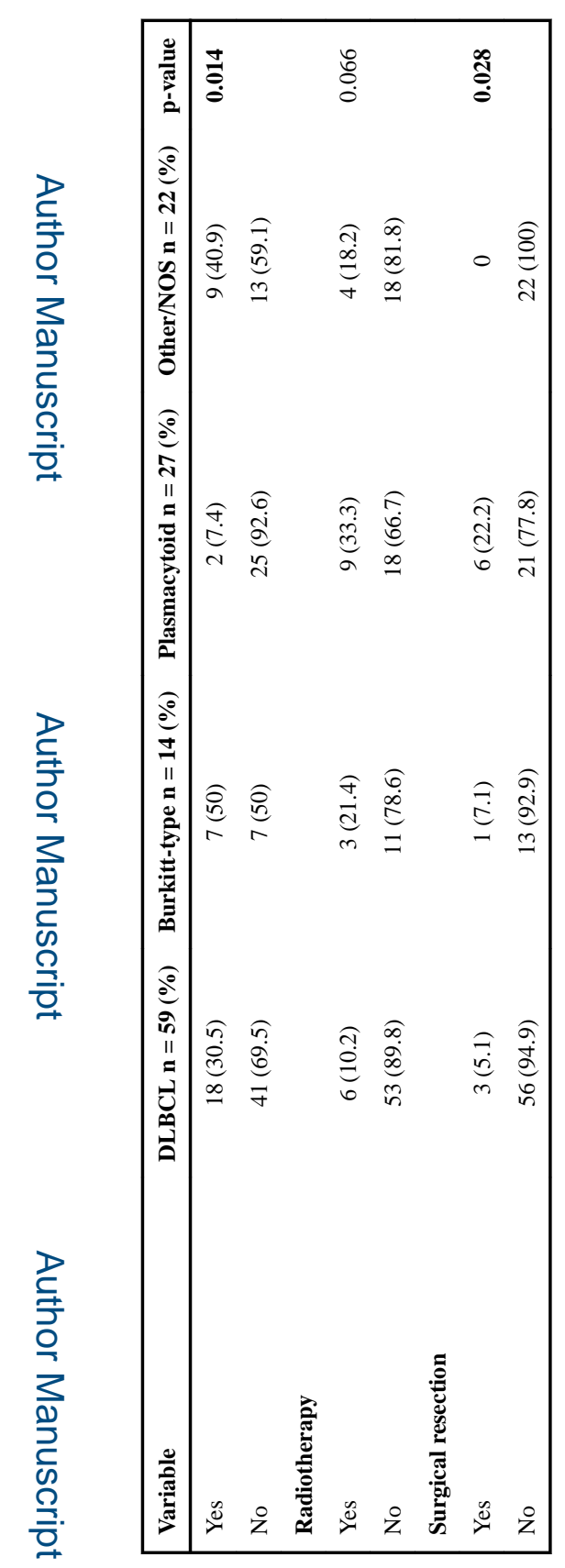

로을

Cancer: Author manuscript; available in PMC 2019 June 01. 


\section{Table 4}

Multivariable analysis of overall survival in monomorphic B-cell post-transplant lymphoproliferative disorder. Number of observations in the original data set $=122$. Number of observations used $=111$. Backward selection with an alpha level of removal of 0.10 was used. The following variables were removed from the model: age, sex, calcineurin inhibitors, anti-metabolites, steroids, chemotherapy, rituximab, and surgical resection. Abbreviations: hazard ratio (HR), confidence interval (CI).

\begin{tabular}{rlll}
\hline Covariate & Level & HR (95\% CI) & HR p-value \\
\hline Organ graft & Lung or Small Bowel & $4.83(1.38-16.96)$ & $\mathbf{0 . 0 1 4}$ \\
& Liver or Liver-Kidney or Liver-Small Bowel & $2.77(1.06-7.27)$ & $\mathbf{0 . 0 3 8}$ \\
& Heart or Heart-Lung & $5.57(2.31-13.44)$ & $<\mathbf{0 . 0 0 1}$ \\
& Kidney or Kidney-Pancreas & - & - \\
Reduction of immunosuppression & Yes & $0.44(0.23-0.84)$ & $\mathbf{0 . 0 1 3}$ \\
& No & Ref & - \\
& Yes & $0.41(0.15-1.13)$ & 0.085 \\
& No & - & -
\end{tabular}




\section{Table 5}

Multivariable analysis of overall survival in monomorphic T-cell post-transplant lymphoproliferative disorder. Number of observations in the original data set $=67$. Number of observations used $=45$. Backward selection with an alpha level of removal of 0.10 was used. The following variables were removed from the model: sex, transplanted organs, reduction of immunosuppression, steroids, chemotherapy, rituximab, and surgical resection. Abbreviations: hazard ratio (HR), confidence interval (CI).

\begin{tabular}{rlll}
\hline Covariate & Level & HR $(\mathbf{9 5 \%}$ CI $)$ & HR p-value \\
\hline Calcineurin inhibitor & Tacrolimus & $0.08(0.01-0.76)$ & $\mathbf{0 . 0 2 8}$ \\
& Cyclosporine & $0.33(0.11-1.04)$ & 0.058 \\
& None & Ref & - \\
Anti-metabolite & Mycophenolate mofetil & $1.20(0.13-11.42)$ & 0.875 \\
& Azathioprine & $3.12(1.10-8.86)$ & $\mathbf{0 . 0 3 2}$ \\
& None & Ref & - \\
Radiotherapy & Yes & $0.32(0.11-0.92)$ & $\mathbf{0 . 0 3 4}$ \\
& No & Ref & - \\
Age & - & $0.97(0.94-1.00)$ & 0.079 \\
\hline
\end{tabular}

